\title{
Nonspatial interdimensional attentional capture
}

\author{
TOMOE INUKAI \\ Chukyo University, Nagoya, Japan \\ AND \\ JUN-ICHIRO KaWAHARA AND TAKATSUNE KUMADA \\ National Institute of Advanced Industrial Science and Technology, Tsukuba, Japan
}

\begin{abstract}
Accuracy in identifying a target is impaired by a task-irrelevant singleton distractor even when the target and distractor appear in the same location. However, whether this impairment, known as a nonspatial interdimensional attentional capture, is contingent on a top-down attentional set or determined by stimulus-driven signals from distractors is unclear. To examine whether interdimensional attentional capture is affected by a top-down attentional set, the present study explicitly manipulated observers' search strategies (the singleton detection or feature search modes) and the number of objects consisting of the search items. The results indicated that interdimensional attentional capture occurred even under the feature search mode but that the capture effect decreased under this search mode irrespective of the number of distractors, suggesting that top-down knowledge was effective in modulating nonspatial interdimensional capture.
\end{abstract}

The human visual system is limited in the number of objects it can process simultaneously. To overcome this limitation, the system directs attention selectively to potentially informative events or objects in two ways. First, attention can be allocated in a top-down endogenous way to a particular location. Second, attention can also be directed in a stimulus-driven way by bottom-up signals. In the latter case, when salient items attract attention exogenously, the control is referred to as attentional capture.

Previous studies have shown several factors that capture attention, such as the abrupt onset of new objects (e.g., Yantis \& Hillstrom, 1994; Yantis \& Jonides, 1984) or a luminance change (e.g., Franconeri, Hollingworth, \& Simons, 2005). On the basis of a series of visual search experiments, Theeuwes $(1991,1992)$ proposed that attentional capture occurs purely in a stimulus-driven way by showing that observers could not ignore a salient distractor (singleton) in a task-irrelevant stimulus dimension. In his study (Theeuwes, 1992), the reaction time for discriminating a target (a green circle) embedded among uniform nontargets (green diamonds) was longer when a singleton (a red diamond) was presented, as compared with when no singleton was presented. He suggested that this delay occurs because the attentional focus was diverted exogenously at the location of the singleton before it reached the target location (cross-dimensional attentional capture).

However, top-down knowledge also modulates whether attentional capture occurs when observers are attentionally set to search for a specific target feature (Folk \& Remington, 1998; Folk, Remington, \& Johnston, 1992;
Gibson \& Kelsey, 1998; Lamy \& Egeth, 2003). For example, Bacon and Egeth (1994) introduced two different strategies, singleton detection and feature search modes, to interpret Theeuwes's (1992) findings of crossdimensional capture. They suggested that the attentional capture observed in Theeuwes's (1992) study indicated that participants adopted a strategy of searching for an oddball among homogeneous nontargets, rather than demonstrating their inability to ignore the salient distractors. When Bacon and Egeth embedded a circular target among heterogeneously shaped nontargets, the capture by the red item disappeared, implying that the heterogeneity of nontargets forced the observers to search for a specific shape-related feature. In other words, the participants had to adopt the feature search mode, because the singleton detection mode was no longer available.

This notion of search modes did not settle the debate over the locus of control in attentional deployment. Theeuwes (2004) demonstrated that cross-dimensional capture occurred even with the same display as that used by Bacon and Egeth (1994) when the relative salience of the singleton was strengthened by increasing the number of nontargets. This finding suggests that attentional deployment depends critically on the saliency of the singleton. Recently, however, Leber and Egeth (2006) used a novel approach involving persistence in the task set and provided evidence that contradicted Theeuwes's (2004) conclusion. They found that top-down knowledge of target features eliminated attentional capture even when the relative salience of the singleton was increased by the high density of nontargets. Therefore, the question of whether

J. Kawahara, jun.kawahara@aist.go.jp 
top-down knowledge preempts the deployment of spatial attention on the basis of salience remains unsettled.

Using a different procedure, Folk, Leber, and Egeth (2002) showed that top-down knowledge could override bottom-up signals from salient singletons. When observers identify a target letter in a rapid stream of nontarget letters, the set of observers determines attentional capture. In their study, a unique red or green peripheral item (distractor) was presented briefly before the onset of the target. One group of observers searched for a target of a specific color among heterogeneous nontargets (the feature search mode), and the other group of observers searched for an oddball color target among homogeneous nontargets (the singleton detection mode). For the feature search, the presence of a distractor sharing the same color as the target impaired the identification accuracy of the observers identifying red target letters. However, for the singleton detection observers, who were identifying colored oddball target letters, the presence of distractor items impaired accuracy regardless of their colors. These results indicate that top-down attentional control settings based on the feature search mode can eliminate disruptions due to distractor items.

Other studies have also reported the effect of salience when participants used the feature search mode (Lamy, Bar-Anan, Egeth, \& Carmel, 2006; Lamy, Leber, \& Egeth, 2004). For example, Lamy et al. (2004) extended Folk et al.'s (2002) paradigm and manipulated the relative salience of a peripheral singleton by embedding it in either homogeneous or heterogeneous nontargets. Lamy et al. (2004) found evidence of attentional capture by the peripheral distractor that shared the same color with the target, irrespective of the relative salience of the distractor. This finding is inconsistent with the purely bottom-up view (Theeuwes, 1992, 2004), in that attentional capture was not determined merely by relative stimulus salience under the feature search mode.

The literature reviewed above indicates that, in general, top-down knowledge seems to affect attentional capture insofar as observers' search modes are appropriately controlled. It is, however, important to note that studies on attentional capture differ in terms of the following two major aspects: spatial versus nonspatial capture and cross- versus within-dimensional capture (Table 1). Because the literature reviewed thus far has involved spatial attentional capture, in which the target and the distractor were spatially displaced, it is unclear whether the same principle applies to nonspatial capture, in which two critical items appear in the same spatial location. As was dis- cussed below, two studies have examined nonspatial capture. However, one addressed within-dimensional capture (Folk, Leber, \& Egeth, 2008). Although the other (Dalton \& Lavie, 2006) focused on cross-dimensional capture, it did not compare the effect of search mode. Therefore, we need to investigate the bottom right quadrant of Table 1 to fully understand the effect of top-down knowledge on attentional capture. Thus, the purpose of the present study was to examine the phenomenon and time course of crossdimensional nonspatial capture in the singleton detection and feature search modes.

Before describing the present experiments, we will briefly summarize the literature on nonspatial capture (the bottom row of Table 1). Folk et al. (2008) initially examined whether attentional capture would occur in the absence of a spatial deployment of attentional focus. They found that the same principle that applied to the case of spatial attentional capture also applied to nonspatial attentional capture. That is, as long as the target and the distractors belong to the same stimulus dimension, such as color, and observers use the singleton detection mode, a singleton causes attentional capture (the intradimensional attentional capture); this does not occur, however, if observers use the feature search mode. However, it is unclear whether the same is true when the singleton distractor belongs to a different stimulus dimension. Second, in terms of cross-dimensional capture, Dalton and Lavie (2006) demonstrated that nonspatial attention was captured by singleton distractors. Their observers searched for a size oddball among a stream of medium-sized nontarget letters and reported whether the target size was small or large. The time taken to discriminate target size was longer when the color singleton was presented immediately before the target than when no color singleton appeared in the stream. This finding suggests that deployment of nonspatial attention is driven by bottom-up control, because the attentional control settings for specifying a target dimension to be searched did not modulate nonspatial attentional capture. Because search strategies were not compared in Dalton and Lavie's study, in which participants seemed to search for a size oddball with the singleton detection mode, the color singleton might have failed to attract nonspatial attention if the participants adopted the feature search mode, in that they were attentionally set to search for a specific target feature (e.g., large).

Thus, more information is required to fully understand the nature of attentional capture in singleton detection, as compared with the feature search mode. The present study was designed to examine this issue. We explicitly manipu-

Table 1

Summary of the Literature Regarding Within-/Cross-Dimensional Spatial/Nonspatial Capture

\begin{tabular}{|c|c|c|}
\hline & Within-Dimensional Capture & Cross-Dimensional Capture \\
\hline Spatial & $\begin{array}{l}\text { Folk, Leber, and Egeth (2002) } \\
\text { Lamy, Leber, and Egeth (2004) } \\
\text { Lamy, Bar-Anan, Egeth, and Carmel (2006) }\end{array}$ & $\begin{array}{l}\text { Theeuwes (1992)* } \\
\text { Bacon and Egeth (1994) }\end{array}$ \\
\hline Nonspatial & Folk, Leber, and Egeth (2008) & $\begin{array}{l}\text { Dalton and Lavie }(2006)^{*} \\
\text { The present study }\end{array}$ \\
\hline
\end{tabular}

*Studies that did not explicitly manipulate observers' search strategies. 
lated observers' search strategies to determine whether top-down knowledge about a specific target feature or a specific target dimension would eliminate nonspatial attentional capture. If the former were the case, attentional capture would be observed under the singleton detection mode, but if the latter were the case, nonspatial capture would be absent under the two modes. In contrast, if neither were the case, nonspatial attentional capture would be observed regardless of the search mode, suggesting that nonspatial attention was governed by bottom-up signals. In addition, we varied the distractor-target temporal lag to observe the temporal profile of attentional capture. Furthermore, to rule out the possibility that the presence of an additional singleton simply had delayed the responses, rather than captured attention, ${ }^{1}$ in Dalton and Lavie's (2006) study in which response time was used as the dependent variable, we measured accuracy, instead of reaction time, for participants' performances in detecting a singleton.

\section{EXPERIMENT 1A}

In Experiment 1A, we examined whether top-down knowledge of the target would eliminate nonspatial attentional capture by a singleton distractor that differed from a target in a critical dimension when varying search strategies were employed. We presented a rapid stream of white tilted alphabet letters. One group of observers searched for an odd target (the singleton detection group) in a stream containing an orientation singleton (a target letter) with a color distractor (either a red letter or a digit preceding the target). Under this condition, the observers' task was to identify an oddly tilted letter while ignoring a color singleton distractor, if any. The other group of observers searched for a target tilted according to a specific orientation (the feature search group). The distractor was a singleton in terms of a task-irrelevant dimension (color). We hypothesized that cross-dimensional capture would occur in the singleton detection group. Because the capture effect was observed by Dalton and Lavie (2006), using the size and color dimensions, a similar finding in this study would generalize their results. The feature search group would provide critical information: If top-down knowledge about a target feature cannot modulate attentional deployment, attentional capture would ensue when observers adopted the feature search mode. Such a result would imply that the availability of top-down knowledge (Folk et al., 2008) is limited to cases of within-dimensional capture. On the other hand, if top-down knowledge governs attentional deployment, attentional capture would be eliminated (or at least reduced) if this knowledge contributed to attentional control to at least some degree.

In addition, we explored the extent to which the relative salience of the singleton distractor would affect the magnitude of nonspatial attentional capture by manipulating the shape category of singleton distractors, because we did not know whether categorical differences would interact with nonspatial attentional capture. The color singleton distractor could be drawn from the same letter category as a target or from a different category (i.e., digit). We sur- mised that the relative salience of the singleton would be higher when it was a digit than when it was a letter. If the amount of attentional capture were governed only by the relative salience of the distractor (e.g., Theeuwes, 1992), a digit singleton that deviated from other letter stimuli in terms of category would produce a stronger capture effect.

\section{Method}

Participants. Forty-four undergraduate students from the National Institute of Advanced Industrial Science and Technology (AIST; Tsukuba, Japan) participant pool participated for pay. All reported normal or corrected-to-normal visual acuity and normal color vision.

Apparatus. The same apparatus was used in all the experiments. All the stimuli were presented on a CRT monitor (GDM-19PS, Sony), controlled by a PC/AT-compatible computer. Responses were collected via a keyboard connected to the computer.

Stimuli. The stimuli consisted of the letters of the alphabet, except $\mathrm{H}, \mathrm{I}, \mathrm{M}, \mathrm{N}, \mathrm{O}, \mathrm{Q}, \mathrm{S}, \mathrm{W}, \mathrm{X}$, and $\mathrm{Z}$, and digits ranging from 1 to 7 , except 6 , because these items were point symmetric or confusable with other letters or digits when rotated. The height and width of the characters subtended approximately $1^{\circ} \times 1^{\circ}$, with a $0.1^{\circ}$ stroke. The stimuli were white or red on a black background. The alphabet letters and digits were oriented $45^{\circ}, 135^{\circ}, 225^{\circ}$, or $315^{\circ}$ from the upright orientation in a clockwise direction.

Design and Procedure. The observers were presented with one of two displays, according to their group assignment. The 22 observers in the singleton detection group identified an oddly tilted letter among homogeneously tilted nontargets (see Figure 1A). The orientation of the target letter on each trial was randomly selected from a set of four possible angles $\left(45^{\circ}, 135^{\circ}, 225^{\circ}\right.$, or $\left.315^{\circ}\right)$, and all the nontarget letters had the same orientation, chosen from among the remaining angles. The 22 observers in the feature search group identified a letter tilted according to a specific orientation among nontargets with various tilts. The orientation of the target was either $45^{\circ}$ or $315^{\circ}$. Half of the feature search observers searched for a target tilted by $45^{\circ}$, and the other half searched for a target tilted by $315^{\circ}$. The orientations of the nontarget letters were chosen from a set of three orientations differing from that of the target, and the same orientation was not used in successive frames. When the orientation of the target was $45^{\circ}$, the orientation of the nontarget letters was $135^{\circ}$, $225^{\circ}$, or $315^{\circ}$. When the orientation of the target was $315^{\circ}$, the orientation of the nontarget letters was $45^{\circ}, 135^{\circ}$, or $225^{\circ}$.

The observers viewed the monitor from a distance of approximately $60 \mathrm{~cm}$ in a normally lit room. Each trial began with the presentation of a fixation point for $500 \mathrm{msec}$ at the center of the monitor. Then the stimuli were presented in rapid succession at the center of the screen. Each stimulus frame was presented for $100 \mathrm{msec}$, and blanks were not inserted before the next frame, yielding a presentation rate of 10 stimuli/sec. A target letter was randomly chosen from the alphabet letters, and each nontarget letter was randomly chosen from the remaining letters, without selecting the same letter for successive frames. The number of nontarget letters preceding the distractor varied randomly from 6 to 11 across trials. The target was consistently followed by a mask stimulus that consisted of some random line segments.

One session consisted of three distractor conditions. In the nodistractor condition, all the nontarget letters were white. In the digit condition, one of the nontarget letters that preceded a target was replaced by a red digit. In the letter condition, one of the nontarget letters was replaced by a red nontarget letter. In the digit and letter conditions, a target appeared at one of five lags $(100,200,300,500$, or $700 \mathrm{msec}$ ) after a singleton distractor. These were referred to as lag 1 , lag 2 , lag 3 , lag 5 , and lag 7 , respectively. The nontargets continued to be displayed during the lag.

The observers were given verbal and written instructions about the nature of the stimuli and the procedures of the task. The observers were asked to identify the target letter while ignoring color 

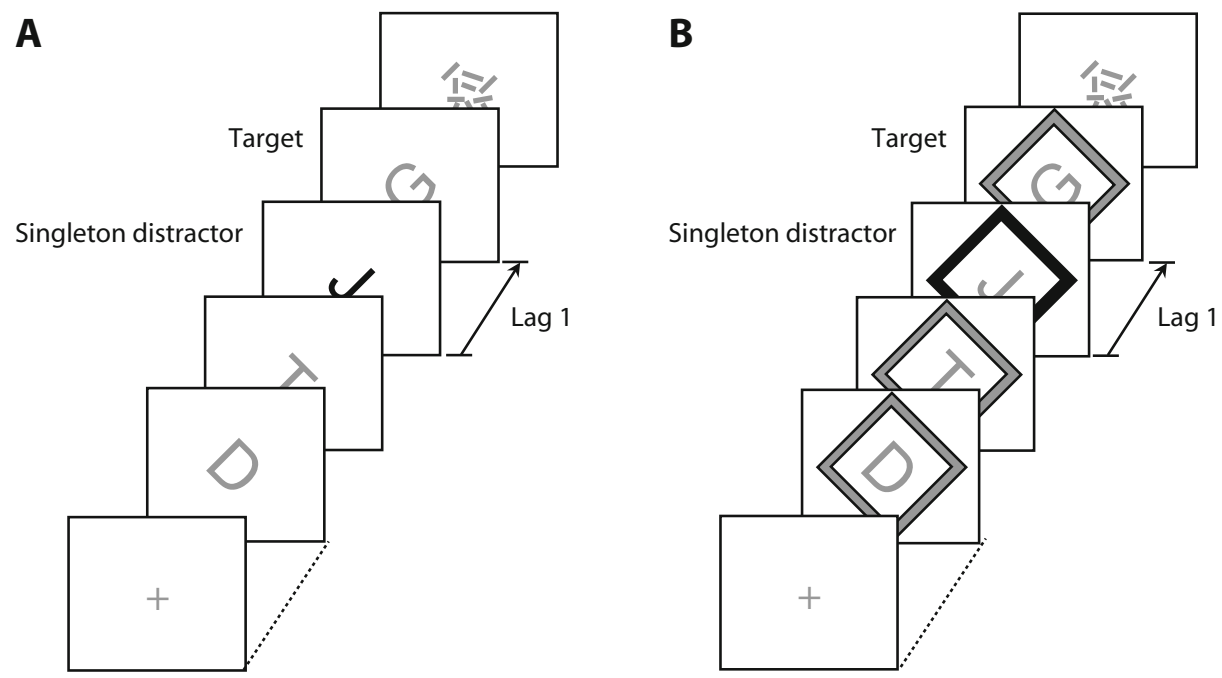

Figure 1. Schematic diagrams of the stimuli and sequence of events in Experiment $1 \mathrm{~A}(\mathrm{~A})$ and Experiment 2 (B). Gray letters and outline frames were actually white, and the black letter and outline frame were red. Panel A describes a case in which a distractor frame containing a red letter appears at lag 1 (immediately before the onset of the target). Panel $B$ describes a case in which a distractor frame containing a nontarget letter with a red outline appears at lag 1.

singletons. At the end of a trial, they typed the key corresponding to the target letter and then pressed the space bar at their own pace to initiate the next trial. The session consisted of 30 practice trials and 270 experimental trials. The number of trials for the three distractor conditions was equal ( 90 trials in each condition). In the letter and digit conditions, equal numbers of lag conditions were presented. These conditions were presented in random order. If the observers needed to take a break during the session, they took a break at their own discretion before starting the next trial.

\section{Results}

Figure 2 shows the mean percentages of correct letter identification under the singleton detection and feature search modes, plotted as a function of lags in the digit and letter conditions, along with the mean scores in the nodistractor condition. First, a three-way ANOVA was performed, with search mode (singleton detection and feature search) as a between-subjects variable and distractor condition (the digit and letter conditions) and lag (100, 200, 300,500 , and $700 \mathrm{msec}$ ) as within-subjects variables. ${ }^{2}$ The main effect of lag was significant $[F(4,168)=13.82$, $p<.001]$, and the main effect of distractor condition was marginally significant $[F(1,42)=3.56, p=.066]$. The interaction between search mode and lag approached significance $[F(4,168)=2.02, p=.093]$. Multiple comparisons with Ryan's (1960) tests revealed that the accuracy at lag 1 was significantly lower than those at the remaining lags $(p<.05)$; no significant differences were observed in any other pairs of comparison.

Next, $t$ tests between accuracy in the no-distractor condition and that at each lag in the digit and letter conditions were conducted for each group. For the singleton detection group, accuracy in the digit and letter conditions at lag 1 was significantly lower than that in the no-distractor condition $[t(21)=5.46, p<.01$, and $t(21)=5.12, p<$ .01 , respectively]. The rates of accurate responses at lag 3 in the digit condition and at lag 5 in the letter condition were marginally lower than were those in the no-distractor condition $[t(21)=1.98$ and $t(21)=1.81$, respectively]. For the feature search group, accuracy in the digit and letter conditions at lag 1 was significantly lower than that in the no-distractor condition $[t(21)=3.04, p<.01$, and $t(21)=3.96, p<.01$, respectively]. Accuracy at lag 5 in the digit condition was significantly lower than that in the no-distractor condition $[t(21)=2.11, p<.05]$. These results indicate that target identification decreased when the singleton distractors appeared immediately before target presentation, regardless of singleton distractor types.

\section{Discussion}

Target identification was impaired whenever the target was preceded by a color singleton, as compared with when no singleton was presented. Such impairment, termed nonspatial interdimensional attentional capture, was obtained under both search modes. This result demonstrated two points. First, nonspatial attention was captured by the singleton distractors under the singleton detection mode when the singleton distractor was defined by a dimension different from that of the target. Second, a singleton in a task-irrelevant dimension impaired search even when the observers were required to adopt the feature search mode. In other words, the deployment of attention under the feature search mode was not exclusively top-down control in certain conditions in the present experiment. However, top-down knowledge did play a role in deploying nonspatial attention, as evidenced by a marginally significant interaction between search mode and lag. Therefore, the present results demonstrated cross-dimensional nonspatial attentional capture, as well as a suggestion of a top-down modulation effect. We will return to this issue in Experiment 2.

The magnitude of nonspatial attentional capture in the letter condition was comparable to that in the digit condi- 


\section{Singleton Detection Mode}

Feature Search Mode

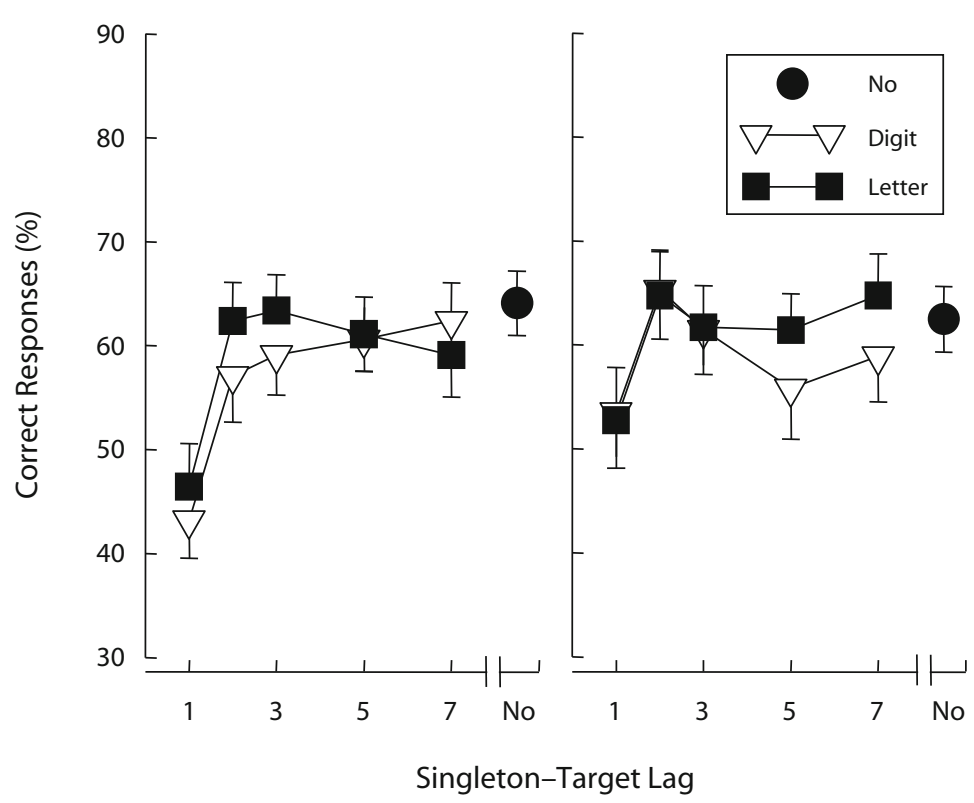

Figure 2. Mean percentages of correct target identifications as a function of lag in the digit and letter conditions, along with the mean score in the nodistractor condition, under the singleton detection and feature search modes in Experiment 1A. Bars indicate standard errors.

tion. This suggests that the observed capture effect was driven by the uniqueness of dimension, indicating that the singleton distractor and the symbolic differences between the letters and digit did not contribute to the effect. The manipulation of the letter/digit category in the present experiment did not affect the results, because the letter-digit distinction did not represent a sufficiently salient difference in the present context. Therefore, in the service of simplicity, we collapsed the distractor conditions (the digit and letter conditions) in the following analyses; this distinction was not further examined, because it had no effect.

The results for the singleton detection group were consistent with those reported by Dalton and Lavie (2006) in the study of reaction time, in that nonspatial interdimensional attentional capture occurred when observers adopted the singleton detection mode. Interestingly, nonspatial capture was also evident in the results obtained from the feature search group. At first glance, this result seems inconsistent with the notion that the kind of singleton distractors that capture attention depends on the sets of observers (Folk et al., 2008; Folk \& Remington, 1998), since it suggests that the contingency principle of attentional capture might be applied only in cases of withindimensional nonspatial capture. Any such interpretation, however, must be treated with caution, for the following two reasons. First, the present results revealed a marginally significant interaction between search mode and lag, implying top-down modulation. Second, the number of objects that composed the singleton distractor differed across the present and Folk et al.'s (2008) experiments; the present study used one object as a singleton distractor (i.e., a colored singleton letter or digit), whereas the study conducted by Folk et al. (2008) used two objects (i.e., a letter framed by an outline). If the number of objects consisting of the singleton represented a critical determinant of whether attentional capture by singletons in a different dimension would emerge, the capture effect would not have appeared in the present study in the conditions in which the singleton distractor consisted of two objects.

Before we address this question in Experiment 2, one concern must first be examined and dismissed. The concern is that the impairment obtained in Experiment 1A might have reflected a forward masking by the distractor, rather than attentional capture. Because the salient singleton distractors were presented immediately before the target at the same location in the digit and letter conditions, this could cause the masking effect. We examined this possibility in Experiment 1B.

\section{EXPERIMENT 1B}

To test the possibility that the impairment in target identification in Experiment 1A was caused by forward masking, we replaced some white nontargets before a singleton with red nontargets under the singleton detection mode. By our doing this, the red distractors were no longer salient singletons with respect to the color dimension. Therefore, if the impairment were due to attentional capture, no impairment would be obtained in Experiment 1B. In this case, identification accuracy would remain the same across the lag. In contrast, if the impairment were due to forward masking, the target identification should 
be impaired when red letters were presented close in time to targets, regardless of whether they were or were not singletons. In this case, a pattern of results similar to that in Experiment 1A would be observed.

\section{Method}

Participants. Twenty experimentally naive undergraduate students from the AIST participant pool participated for pay. All reported normal or corrected-to-normal visual acuity and normal color vision.

Apparatus, Stimuli, and Procedure. The apparatus, stimuli, and procedure were identical to those used for the singleton detection group in Experiment 1A, with the following exceptions. The letter condition was not included. In the digit condition, half of the nontarget letters preceding a digit singleton were changed to red, yielding an alternation of the colors of nontarget letters before the nominal singleton. Note that a digit singleton was not a color oddball in this experiment, because the original red item in Experiment 1A was no longer unique as the color item. After the nominal singleton, all the items were white. The stimulus stream started with a red letter on half of the trials. The no-distractor condition was the same as that in Experiment 1A. The lag between the digit distractor and the target was 100,200 , or $500 \mathrm{msec}$. A session consisted of 30 practice trials and 108 experimental trials.

\section{Results and Discussion}

Figure 3 shows the percentage of correct identifications in the digit condition as a function of lag and the mean score in the no-distractor condition. In the digit condition, a one-way ANOVA with lag as a within-subjects factor indicated no significant main effect of $\operatorname{lag}[F(2,38)<1$, n.s.]. The analysis revealed that performance was unaffected by the digit singleton when it appeared in the heterogeneous color of distractors. To compare accuracy in the no-distractor condition with that in the digit condition,

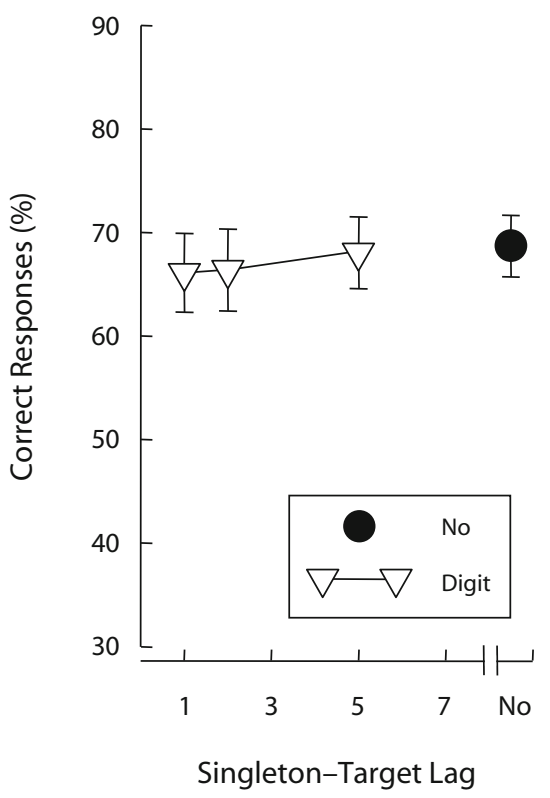

Figure 3. Mean percentages of correct target identifications as a function of lag in the digit condition, along with the mean score in the no-distractor condition in Experiment 1B. Bars indicate standard errors. a $t$ test was conducted for accuracy in the no-distractor condition and the digit condition at each lag, again yielding no significant difference between the two conditions for any pairs $[t \mathrm{~s}(19)<0.87]$.

The result showed that the impairment of target identification at lag 1 found in Experiment $1 \mathrm{~A}$ was not due to forward masking of the target by the singleton distractor. If the impairment of target identification found in Experiment $1 \mathrm{~A}$ had been due to forward masking, a similar impairment should have been obtained in this experiment, because the temporal relationship between the (nominal) singleton and the target was identical across Experiments $1 \mathrm{~A}$ and 1B. In fact, no impairment was found in Experiment $1 \mathrm{~B}$, suggesting that forward masking did not occur. More important, when the distractors were no longer singletons, the observers could ignore them. Therefore, we concluded that the impairment obtained in Experiment 1A was caused by nonspatial attentional capture, rather than by forward masking.

The results thus far suggest that cross-dimensional capture might differ from spatial capture. In attempting to examine this issue further, we suggest that the present study differed from the previous study (Folk et al., 2008) with respect to more than the issue of within- versus betweendimensional capture; that is, we suggest they also differed with regard to whether one or two objects were used as a target. Experiment 2 examined this issue.

\section{EXPERIMENT 2}

In this experiment, we examined whether the difference in the number of objects consisting of a target item might account for the different patterns of results observed with respect to cross-dimensional capture in the present study and within-dimensional capture in previous experiments (Folk et al., 2008). To this end, we employed displays similar to those used by Folk et al. (2008), in that all the stimuli were outlined by a frame. Experiment 2 was designed to investigate whether a singleton distractor that was composed of two objects would cause nonspatial interdimensional attentional capture under the two search modes. The observers in the singleton detection group identified an oddly tilted letter with a white outline frame, while ignoring a nontarget letter with a red frame. The observers in the feature search group identified a letter tilted in a specific orientation with a white frame, while ignoring a nontarget letter with a red frame.

\section{Method}

The apparatus, stimuli, and procedure were identical to those in Experiment 1A, with the following exceptions. As is illustrated in Figure $1 \mathrm{~B}$, each stimulus was surrounded by a white outline frame that measured approximately $1.6^{\circ} \times 1.6^{\circ}$, with a $0.1^{\circ}$ stroke, except for one of the nontargets (a letter or digit). This nontarget was surrounded by a red outline frame and was designated as a singleton distractor. The target was also presented with a white frame. Fortytwo experimentally naive undergraduate students from the AIST participant pool participated for pay. All reported normal or correctedto-normal visual acuity and normal color vision. Equal numbers of participants were randomly assigned to the singleton detection and the feature search groups. The latter group was further divided into 
two groups; 11 observers identified a target tilted by $45^{\circ}$, and the remaining 10 observers identified a target tilted by $315^{\circ}$.

\section{Results}

Figure 4 shows the mean percentages of correctly identified letters for the singleton detection and feature search groups, plotted as a function of lags in the distractor condition, along with the mean scores in the no-distractor condition. A two-way ANOVA with search mode (the singleton detection and feature search modes) as a between-subjects variable and lag as a withinsubjects variable revealed a significant main effect of lag $[F(4,160)=3.09, p<.05]$. Multiple comparisons by Ryan's (1960) tests revealed that the accuracy at lag 1 was significantly lower than that at lag $7(p<.05)$. No significant differences were observed in any other pairs of comparisons. The main effect of search mode approached significance $[F(1,40)=3.75, p=.06]$. The interaction between search mode and lag was not significant $[F(1,160)<1$, n.s. $]$.

Next, a $t$ test was conducted between accuracy in the no-distractor condition and accuracy at each lag in the distractor condition. When the observers identified an oddly tilted letter (i.e., the singleton detection mode), the accuracy rates in the distractor condition at all lags except lag 7 were significantly lower than those in the no-distractor condition $[t(20)=3.2, p<.05 ; t(20)=3.66, p<.01$; $t(20)=3.05, p<.05$; and $t(20)=2.28, p<.05$, respectively]. When the observers identified a specific tilted letter (i.e., the feature search mode), accuracy rates in the distractor condition at lag 1 were significantly lower than were those in the no-distractor condition $[t(20)=3.16$, $p<.05]$.

\section{Discussion}

Target identification was impaired when a color singleton distractor preceded the target under the singleton detection and feature search modes when the singleton distractor consisted of two objects (a nontarget letter or digit and its surrounding frame). This pattern of results was consistent with that obtained in Experiment 1A, but it was inconsistent with that reported by Folk et al. (2008), in that top-down knowledge of target features did not eliminate nonspatial attentional capture. In terms of contingent capture, these results suggest that the general principle that attentional capture does not occur when the defining feature of the singleton distractor differs from the observers' attentional set did not completely apply to the cases tested in the present study, in which a cross-dimensional singleton was used, even when the distractor consisted of two objects. It is, however, important to note that the effect of search mode approached significance, as was the case in Experiment 1A. Therefore, search mode seemed to exert a weak effect in the present study.

To test this possibility, we submitted the results of Experiments $1 \mathrm{~A}$ and 2 to a between-experiments ANOVA on lag 1 scores, with singleton (present vs. absent) as the within-subjects factor and number of objects (one vs. two) and search mode (singleton detection vs. feature search) as the between-subjects factors. If search mode modulated nonspatial attentional deployment (i.e., if top-down knowledge based on the feature search mode decreased

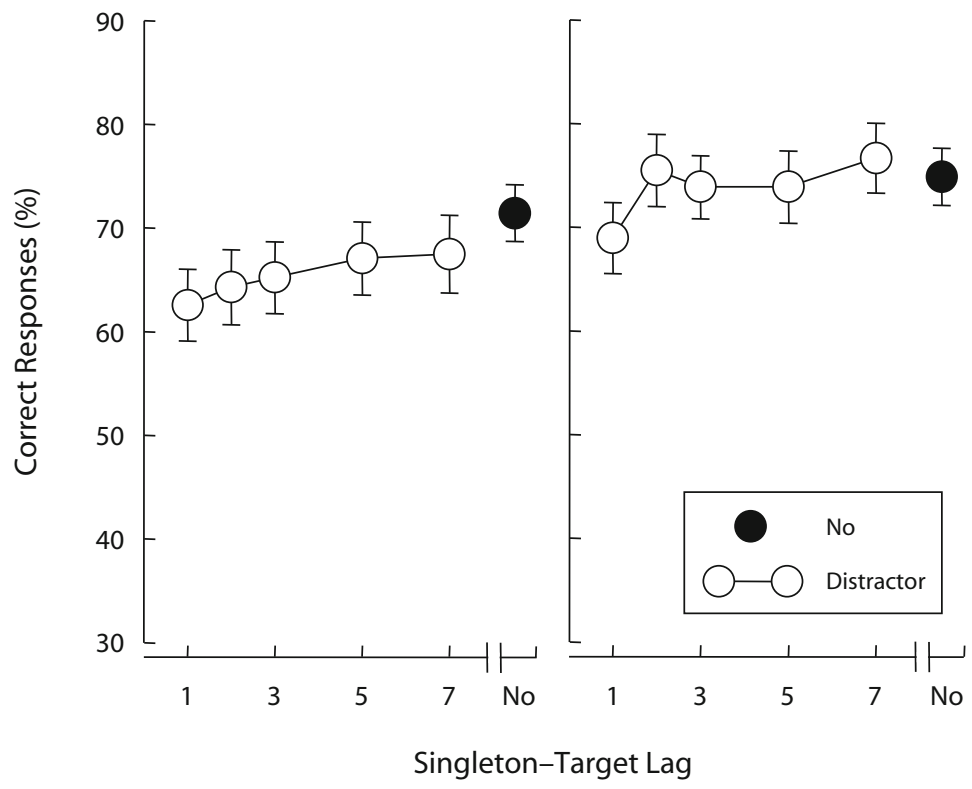

Figure 4. Mean percentages of correct target identifications as a function of lag in the distractor condition, along with the mean score in the no-distractor condition, under the singleton detection and feature search modes in Experiment 2. Bars indicate standard errors. 
the capture effect), a three-way interaction would be obtained. The ANOVA revealed significant main effects of number of objects $[F(1,82)=18.75, p<.001]$ and singleton $[F(1,82)=73.56, p<.001]$. The interactions between search mode and singleton $[F(1,82)=6.70, p<.05]$ and number of objects and singleton $[F(1,82)=7.44, p<$ $.01]$ were also significant. Importantly, the three-way interaction did not reach significance $[F(1,82)=1.96, p=$ .16]. Therefore, the present results suggest that the effect of search mode was quantitative, insofar as the featuresearch mode weakened capture without eliminating it.

\section{GENERAL DISCUSSION}

In the present study, we investigated cross-dimensional nonspatial capture by explicitly controlling observers' search modes while examining the temporal course of the effect. The study contributes the following new knowledge to the literature. First, the studies of cross-dimensional capture comparing the singleton detection and feature search modes have examined only spatial capture (e.g., Bacon \& Egeth, 1994; Theeuwes, 1992). Studies comparing the two search modes with regard to nonspatial capture have addressed only within-dimensional capture (e.g., Folk et al., 2008). In addition, the study of nonspatial cross-dimensional capture that omitted comparisons of the two search modes (Dalton \& Lavie, 2006) did not involve manipulation of the temporal lag between the distractor and target.

Thus, the present results clarified three issues regarding the nature of the deployment of nonspatial attention. First, the present study showed that nonspatial interdimensional attentional capture occurred when observers used the singleton detection mode. The same results emerged when they used the feature search mode. These results confirmed the robustness of the interdimensional attentional capture effect and generalized Dalton and Lavie's (2006) findings, in that the effect is not limited to the stimulus dimension (i.e., size and color) that they used in their study. Second, the present study revealed the temporal dynamics of interdimensional attentional capture, in that the capture attributed to a cross-dimensional singleton lasted a relatively short period, up to lag 1 (100 $\mathrm{msec})$.

Finally and most important, the capture effect decreased under the singleton detection mode, as evidenced by the interaction between search mode and singleton in the between-experiments analysis. However, the capture did not disappear under the feature search mode. In this sense, search mode did not alter search in a qualitative way. In other words, the effect of search mode was quantitative insofar as the feature search mode weakened capture without eliminating it. This argument is consistent with the two observations emerging from the graphs depicting the results of Experiments $1 \mathrm{~A}$ and 2. First, the addition of a frame (Experiment 2) reduced capture by the irrelevant distractor. Indeed, when the search modes were compared, the reductions in accuracy at lag 1 were approximately $20 \%$ and $10 \%$ in the one- and two-object conditions, respectively, when the target feature was unknown (the singleton detection modes in Experiment 1A vs. 2). A difference in the same direction was also apparent when the target feature was known (the feature search modes in Experiment $1 \mathrm{~A}$ vs. 2): approximately $10 \%$ versus $6 \%$ if the two distractor conditions were averaged. Second, the availability of feature information reduced capture (singleton detection vs. feature search mode) from approximately $20 \%$ to $10 \%$ when one object was involved (Experiment 1A) and from approximately $10 \%$ to $6 \%$ when two objects were involved (Experiment 2). Therefore, on the basis of this observation, one could argue that the availability of top-down information and the number of objects played roles in all the experiments.

We do not know why the number of objects affected the magnitude of cross-dimensional attentional capture in the present study. Specifically, attentional capture decreased when the distractor consisted of two objects (Experiment 2), relative to when it consisted of one object (Experiment 1A). It is possible that this finding derived from the greater cost to the visual system when the same information was conveyed by two versus a single object (e.g., Duncan, 1984; Kramer, Weber, \& Watson, 1997). When the distractor consisted of one object (Experiment 1A), such a two-object cost would not have obtained, and thus the distractor (the colored item) itself might have caused the visual system to process letter identity (e.g., Ghorashi, Zuvic, Visser, \& Di Lollo, 2003; Maki \& Mebane, 2006). This process might cause a temporary unavailability of identification processing, resulting in a failure of target identification for a subsequently presented target. On the other hand, when the distractor was defined by two objects (i.e., the frame and the letter inside), the two-object cost reduced the impact of the imperativeness with which the visual system processed the letter.

In summary, the present study demonstrated a case in which nonspatial interdimensional attentional capture occurs under the singleton detection and the feature search modes. However, we found that attentional deployment was subject to observers' search modes. When disruptions derive from a task-irrelevant dimension, top-down control settings are available in conjunction with the bottom-up disruptions. In this sense, the present results might contribute to mediating the controversy over the effect of topdown knowledge on attentional capture. Likewise, the present study highlighted the limitations of search mode with respect to nonspatial capture, insofar as the feature search mode weakened capture without eliminating it.

\section{AUTHOR NOTE}

Correspondence concerning this article should be addressed to T. Inukai, c/o J. Kawahara, National Institute of Advanced Industrial Science and Technology, Central 6, 1-1-1 Higashi, Tsukuba 305-8566, Japan (e-mail: jun.kawahara@aist.go.jp).

\section{REFERENCES}

Bacon, W. F., \& Egeth, H. E. (1994). Overriding stimulus-driven attentional capture. Perception \& Psychophysics, 55, 485-496.

Dalton, P., \& Lavie, N. (2006). Temporal attentional capture: Effects of irrelevant singletons on rapid serial visual search. Psychonomic Bulletin \& Review, 13, 881-885.

DunCAN, J. (1984). Selective attention and the organization of visual attention. Journal of Experimental Psychology: General, 113, 501-517. Folk, C. L., Leber, A. B., \& Egeth, H. E. (2002). Made you blink! 
Contingent attentional capture produces a spatial blink. Perception \& Psychophysics, 64, 741-753.

Folk, C. L., Leber, A. B., \& Egeth, H. E. (2008). Top-down control settings and the attentional blink: Evidence for non-spatial contingent capture. Visual Cognition, 16, 616-642.

FolK, C. L., \& Remington, R. [W.] (1998). Selectivity in distraction by irrelevant featural singletons: Evidence for two forms of attentional capture. Journal of Experimental Psychology: Human Perception \& Performance, 24, 847-858.

Folk, C. L., Remington, R. W., \& Johnston, J. C. (1992). Involuntary covert orienting is contingent on attentional control settings. Journal of Experimental Psychology: Human Perception \& Performance, 18, $1030-1044$

Franconeri, S. L., Hollingworth, A., \& Simons, D. J. (2005). Do new objects capture attention? Psychological Science, 16, 275-281.

Ghorashi, S. M., Zuvic, S. M., Visser, T. A., \& Di Lollo, V. (2003). Focal distraction: Spatial shifts of attentional focus are not required for contingent capture. Journal of Experimental Psychology: Human Perception \& Performance, 29, 78-91.

Gibson, B. S., \& Kelsey, E. M. (1998). Stimulus-driven attentional capture is contingent on attentional set for displaywide visual features. Journal of Experimental Psychology: Human Perception \& Performance, 24, 699-706.

Kramer, A. F., Weber, T. A., \& Watson, S. E. (1997). Objects-based attentional selection-Grouped arrays or spatially invariant representations?: Comment on Vecera and Farah (1994). Journal of Experimental Psychology: General, 126, 3-12.

Lamy, D., Bar-Anan, Y., Egeth, H. E., \& Carmel, T. (2006). Effects of top-down guidance and singleton priming on visual search. Psychonomic Bulletin \& Review, 13, 287-293.

Lamy, D., \& Egeth, H. E. (2003). Attentional capture in singletondetection and feature-search modes. Journal of Experimental Psychology: Human Perception \& Performance, 29, 1003-1020.

LAMy, D., Leber, A., \& EgETH, H. E. (2004). Effects of task relevance and stimulus-driven salience in feature-search mode. Journal of Experimental Psychology: Human Perception \& Performance, 30, 1019-1031.

Leber, A. B., \& Egeth, H. E. (2006). It's under control: Top-down search strategies can override attentional capture. Psychonomic Bulletin \& Review, 13, 132-138.

Maki, W. S., \& Mebane, M. W. (2006). Attentional capture triggers an attentional blink. Psychonomic Bulletin \& Review, 13, 125-131.

Ryan, T. A. (1960). Significance tests for multiple comparison of pro- portions, variances, and other statistics. Psychological Bulletin, 57, 318-328.

Theeuwes, J. (1991). Cross-dimensional perceptual selectivity. Perception \& Psychophysics, 50, 184-193.

TheEuwes, J. (1992). Perceptual selectivity for color and form. Perception \& Psychophysics, 51, 599-606.

Theeuwes, J. (2004). Top-down search strategies cannot override attentional capture. Psychonomic Bulletin \& Review, 11, 65-70.

Yantis, S., \& Hillstrom, A. P. (1994). Stimulus-driven attentional capture: Evidence from equiluminant visual objects. Journal of Experimental Psychology: Human Perception \& Performance, 20, 95-107.

YAntis, S., \& Jonides, J. (1984). Abrupt visual onsets and selective attention: Evidence from visual search. Journal of Experimental Psychology: Human Perception \& Performance, 5, 625-638.

\section{NOTES}

1. We thank Dominique Lamy for pointing out these issues.

2 . The results for the singleton detection group involved targets presented according to four possible orientations, whereas those for the feature search group involved targets presented according to two possible orientations. Although there may have been no difference in the relative salience of the four orientations, this conclusion cannot be supported by the data. Therefore, we analyzed the data obtained under the singleton detection mode to confirm that the same result would emerge. We collapsed the digit and letter conditions because there was no significant difference related to this factor. A two-way lag $(100,200,300,500,700 \mathrm{msec}) \times$ orientation $\left(45^{\circ}, 135^{\circ}, 225^{\circ}, 315^{\circ}\right)$ ANOVA showed a main effect of lag $[F(4,84)=10.68, p<.001]$. Multiple comparisons by Ryan's $(1960)$ method revealed that accuracy at lag 1 was lowest $(t \mathrm{~s}>4.82, p \mathrm{~s}<.05)$. No significant differences were observed among comparisons. More important, differences between the four orientations did not reach significance. This indicated that the result obtained under the singleton detection mode was virtually identical to those described above. Although we should use the same orientation of the targets under the singleton detection and feature search modes to control the relative salience of the four orientations strictly, we believe that differences in the numbers of possible target orientations did not represent a critical underpinning for our conclusions. Therefore, we used data obtained from four possible orientations in Experiment 2 to obtain data that were sufficient for analysis.

(Manuscript received April 18, 2008; revision accepted for publication October 31, 2009.) 